\title{
LABORATORIES
}

* Inactive Laboratories.

${ }^{1}$ The $\mathrm{H}^{3}$-Laboratorium of this institute (directed by Klaus Fröhlich) should be ad. dressed separately.

2 This designation Gif supersedes both Sa (Saclay) and Gsy (Gif-sur-Yvette). The only Gsy date list to be published is Gsy I (Coursaget and Le Run, R. IDIOCARBON, v. 8).

${ }^{3}$ From January 1, 1961 the Gro numbers have been replaced by GrN numbers. "New" dates are referred to the NBS oxalic-acid standard.

${ }^{4}$ Early dates from this laboratory were given a code designation that represents the name of the sponsoring institution, c.g. I ( AGS) for American Geographical Society (Heusser, RADIOCARBON SUPPLEMENT, v. 1).

5 Formerly Hazleton Nuclear; code designation HNS has been dropped.

${ }^{6}$ Some dates from this laboratory were published with the code designation $S$ (Pringle and others, 1957, Science, v. 125, p. 69-70).

' See SM.

${ }^{8}$ See Gif.

"Some dates from this laboratory have been published with the code designation RC (Flint and Gale, 1958, AM. JOUR. SCI., v. 256, p. 698-714). The code designation MP published in volume I of the RADIOCARBON SUPPLEMENT (1959, p. 216) has been changed to $S M$ in conformity with the wishes of the laboratory, and is explained by the change of the company's name from Magnolia Petroleum Company to Socony Mobil Oil Company, Inc.

1" Formerly Texas-Bio-Nuclear, then Kaman Instruments. The laboratory is no longer operating.

A IRIZONA

Dr. Paul E. Damon

Laboratory of Geochemistry

Geochronology Department

Tucson, Arizona 85721

ANL ARGONNE NATIONAL LABORATORY

I) r. F. T. Hageman

Argonne National Laboratory

9700 South Cass Avenue

Argonne, Illinois

ANU AUSTRILIAN NATIONAL UNIVERSITY

Mr. H. A. Polach or Dr. J. F. Lovering

Department of Geophysics and Geochemistry

Australian National University

Canberra A.C.T., Australia

B

BERN

Prof. H. Oeschger

Physikalisches Institut

Universität Bern

Siderstrasse 5

Bern, Switzerland

Birm BIRMINGHAM

Prof. F. W. Shotton, F.R.S

Department of Geology, P.O. Box 363

University of Birmingham

Birmingham 15, England

BIn BERLIN

Ing. Günther Kohl

Deutsche Akademie der Wissenschaften zu Berlin

Institut für Ur-und Frühgeschichte

1199 Berlin, Rudower Chaussee 6

Germany 


\section{BRITISH MUSEUM}

Mr. Harold Barker and Mr. Richard Burleigh

Research Laboratory

The British Museum

London, W.C.1, England

\section{BONN BONN}

Dr. H. W. Scharpensecl and F. Pietig

Institut für Bodenkunde

Universitat Bonn

Bonn, Germany

${ }^{*} \mathrm{C} \quad$ CHICAGO

Dr. W. F. Libby

Institute of Geophysics

University of California

Los Angeles, California 90024

*CT CALTECH

Dr. Don M. Yost

Gates and Crellin Laboratories of Chemistry

California Institute of Technology

Pasadena, California 91109

*D DUBLIN

Dr. William A. Watts

Department of Botany

Trinity College

Dublin, Ireland

Dak DAKAR

Dr. Cheikh Anta Diop

Directeur du Laboratoire de

Radiocarbone I.F.A.N.

Université de Dakar

République du Sénégal

$\mathrm{Fr}^{1} \quad$ FREIBERG

Hajo Stechemesser

C-14 Laboratorium

II. Physikalisches Institut

der Bergakademie Freiberg

Leipziger Strasse 17

Freiberg/Sachsen

Germany

FSU FLORIDA STATE

James R. Martin, H. G. Goodell, and D. S. Phelps

Radiocarbon Dating Laboratory

Department of Geology

Florida State University

Tallahassee, Florida 32306

*G GÖTEBORG

Dr. E. Ehn

Laboratory of Nuclear Chemistry

Chalmers Tekniska Högskola

Göteborg, Sweden

GaK

\section{GAKUSHUIN UNIVERSITY}

Prof. K. Kigoshi

Gakushuin University

Mejiro, Toshima-ku

Tokyo, Japan 
Dr. Wi. Mościcki

Polska Akademia Nauk

Instytut Badan Jadrowych

Pracownia Geochronologii Bezwzglednej

Gdansk-Wrzeszcz, ul. Sobicskicgo 18

Poland

Gif $^{2}$

\section{GIF-SUR-YVETTE}

Dr. J. Labeyrie or Mme. G. Delibrias

Laboratoire du Radiocarbone

Centre National de la Recherche Scientifique

9--Gif-sur-Yvette, France

*GL GEOCHRONOLOGICAL LABORATORY

Institute of Archacology

31-34 Gordon Square

London, W.C.1, England

$\mathrm{Gro}^{8}$

GRONINGEN

Dr. J. C. Vogel

Natuurkundig Laboratorium der Rijks-Universiteit

Westersingel 34

Groningen, Netherlands

GSC OTTAWA

Mr. J. A. Lowdon

Radiocarbon Dating Laboratory

Geological Survey of Canada

601 Booth Strect

Ottawa, Ontario, Canada

*Gsy2 GIF-SUR-IVETTE

GU GLASGOW UNIVERSITY

Dr. A. Walton

Department of Chemistry

The University

Glasgow W.2, Scotland

GX

GEOCHRON I.ABORATORIES INC.

Mr. Harold W. Krueger

Geochron Laboratories Inc.

24 Blackstone Street

Cambridge, Mass. 02139

H

HEIDELBERG

Mr. D. Berdau and Dr. K. O. Münnich

C-14 Laboratorium

II Physikalisches Institut der Universitãt

Heidelberg, Philosophenweg 12

West Germany

Hv

HANNOVER

Dr. M. A. Geyh

Niedersächsisches Landesamt

für Bodenforschung

Hannover-Buchholz, Alfred-Bentz-Haus

West Germany

$I^{4}$

ISOTOPES - A TELEDYNE COMPANY

Dr. Eric H. Willis

Isotopes - A Teledyne Company

50 Van Buren Avenue

Westwood, New Jersey 07675 
II $^{5} \quad$ ISOTOPES, INC.

Palo Alto Laboratories

W. R. Schell

Isotopes, Inc., A Teledyne Company

4062 Fabian Street

Palo Alto, California 94303

IRPA INSTITUT ROYAL DU PATRIMOINE IRTISTIQUE.

Anne Nicole Schreurs

Institut Roval du Patrimoine Artistique

1 Parc du Cinquantcnaire

Brussels 4, Belgium

ISGS ILLINOIS STATE GEOLOGICAL SURVEY

Mr. Stephen M. Kim

Section of Analytical Chemistry

Illinois State Geological Survey

Natural Resources Building

Urbana, Illinois 61801

IVIC CARACAS

Dr. M. A. Tamers

Instituto Venezolano de Investigaciones Científicas

Departmento de Química

Apartado 1827

Caracas, Venezuela

K

COPENHAGEN

Dr. Henrik Tauber

Department of Natural Sciences

National Museum

Copenhagen K, Denmark

KI KIEL

Dr. H. Willkomm and Mr. H. Erlenkeuser

Institut für Reine und Angewandte Kernphysik

Universität Kiel

23 Kiel,

Olshausenstrasse 40-60

Germany

KN KÖLN

Dr. J. Freundlich, Mr. H. H. Eipper

Institut für Ur-und Frühgeschichte der Universität

$\mathrm{C}^{14}$-Laboratorium

Köln (Cologne), Weyertal 125, W. Germany

L LAMONT

Dr. D. L. Thurber

Lamont Geological Observatory

Columbia University

Palisades, New York 10964

Le

LENINGRAD

Radiocarbon Laboratory

Institute of Archaeology

Dvortsovaya 18

Leningrad, D-41, USSR

LJ

\author{
UNIVERSITY OF CALIFORNIA, SAN DIEGO \\ Dr. H. E. Suess \\ Scripps Institution of Oceanography \\ University of California, San Diego \\ La Jolla, California 92037
}


LA PLATA

Dr. Horacio Cazencuve

Museo de La Plata

Padeo del Bosque

La Plata, Argentina

Lu LUNI)

Dr. Tage Nilsson and Mr. Sören Håkansson

Radiocarbon Dating Laboratory

University of Lund

Tunavägen 29

Lund, Sweden

HÉVERLE LOUVAIN

Prof. P. C. Capron and Mr. E. Gilot

Centre de Physique Nucléaire

Avenue Cardinal Mercier

Héverlé Louvain, Belgium

Ly UNIVERSITY OF LYON

Mr. J. Evin

Laboratoire de Radiocarbone

Institut de Physique Nucléaire

43, Boulevard du II Novembre 1918

69, Villeurbanne-Lyon, France

M

\section{MICHIGAN}

Dr. James B. Griffin

University Museums Building

The University of Michigan

Ann Arbor, Michigan 48104

${ }^{*} \mathrm{Ma}^{8} \quad$ MANITOBA

MC MONACO

Dr. J. Thommeret or Mr. J. L. Rapaire

Laboratoire de Radioactivité Appliquée

Centre Scientifique de Monaco

Avenue Saint Martin

Monaco

ML MIAMI

Dr. H. G. Östlund

Institute of Marine Science

University of Miami

Miami, Florida 33149

Mo

VERNADSKI INSTITUTE OF GEOCHEMISTRY

Vernadski Institute of Geochemistry

Academy of Sciences of the USSR

Moscow, USSR

Address: Academician A. P. Vinogradow

Vorobevskoye shosse,d.47-A

Moscow, USSR

$\mathrm{MP}^{7}$ MAGNOLIA PETROLEUM

N RIKEN (TOKYO)

Dr. F. Yamasaki

The Institute of Physical and Chemical Research

Bunkyo-ku, Tokyo, Japan

NPL NATIONAL PHYSICAL LABORATORY

Mr. W. J. Callow and Miss G. I. Hassall

Division of Radiation Science

National Physical Laboratory

Teddington, Middlesex, England 


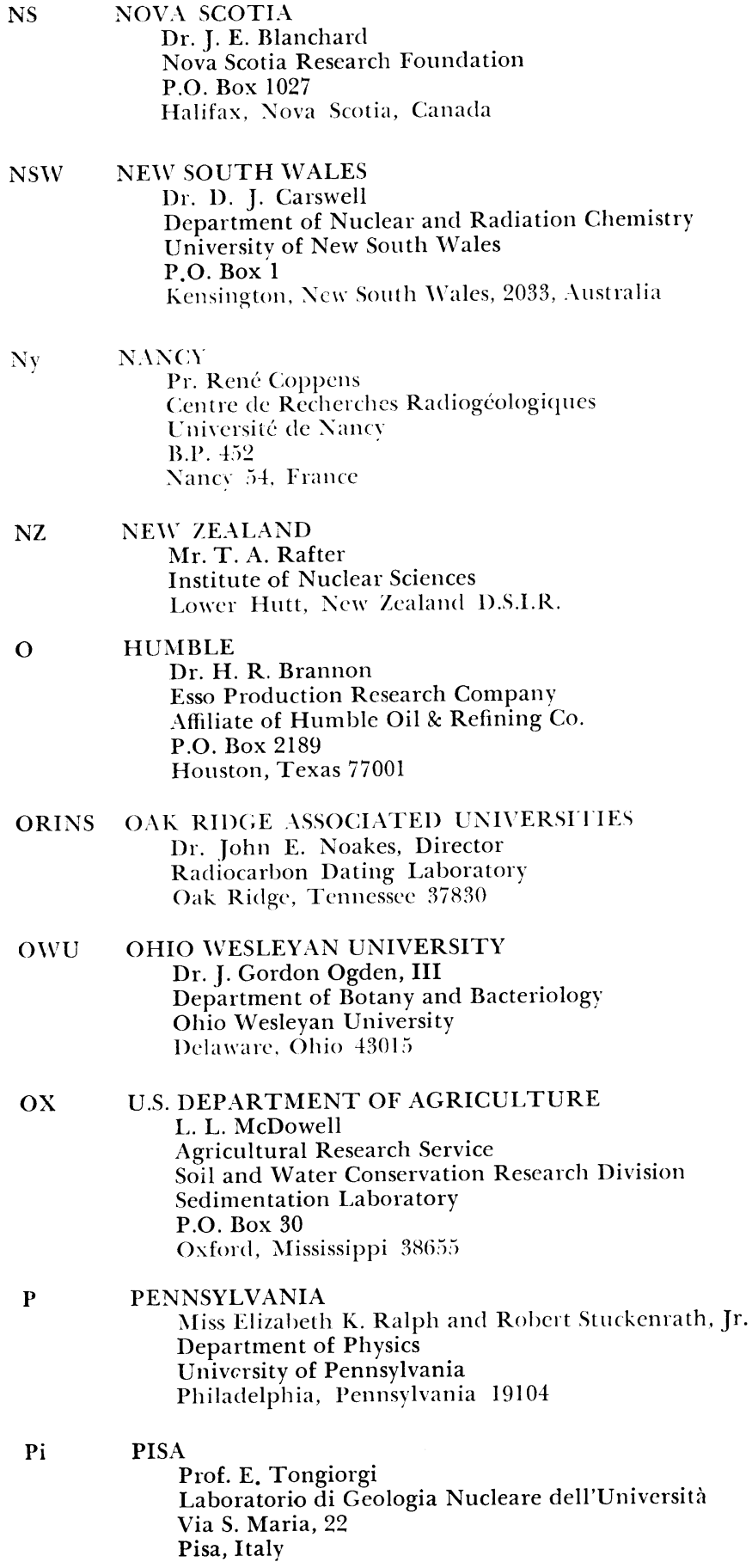


PIC

PACKARD

Dr. Ariel G. Schrodt

Low Level Counting Laboratory

Packard Instrument Co., Inc.

2200 Warrenville Road

Downers Grove, Illinois 60515

Pr

PRAGUE

Alois Dubanský

Laboratory for Isotopes

Geochemistry and Geochronology

Geological Institute

Czechoslovak Academy of Sciences

Prague-8

Na Hrazi 26

$\mathbf{Q}$

CAMBRIDGE

Dr. R. G. West or Dr. V. R. Switsur

University Sub-Department of Quaternary Research

Botany School

Downing Street

Cambridge, England

$\mathbf{R}$

ROME

Dr. F. Bella, Istituto di Fisica

and

Dr. C. Cortesi, Istituto di Geochimica

Radiocarbon Dating Laboratory

University of Rome

Citta Universitaria

Rome, Italy

RI

RADIOCHEMISTRY, INC.

F. M. Sweets

Radiochemistry, Inc., Subsidiary of

The Martin Sweets Co., Inc.

3131 West Market Street

Louisville, Kentucky 40212

S

SASKATCHEWAN

Dr. K. J. McCallum

Department of Chemistry

University of Saskatchewan

Saskatoon, Saskatchewan, Canada

${ }^{*} \mathrm{Sa}^{8} \quad$ SACLAY

Sh SHELL

Dr. E. L. Martin

Shell Development Company

P.O. Box 481

Houston, Texas 77002

SI

SMITHSONIAN INSTITUTION

Dr. W. H. Klein, Director

Radiation Biology Laboratory

Smithsonian Instiution

Washington, D.C. 20560

*SL SHARP LABORATORIES

SM $^{9}$ MOBIL OIL CORPORATION

Dr. H. F. Nelson

Mobil Oil Research and Development Corp.

Field Research Laboratory

P.O. Box 900

Dallas, Texas 7522 
SALISBURY, RHODESIA

Dr. E. R. Swart or Dr. J. G. Sheppard

Gulbenkian Radiocarbon Dating Laboratory

Department of Chemistry

University of Rhodesia

P. Bag $167 \mathrm{H}$

Salisbury, Rhodesia

St

STOCKHOLM

Mr. Lars Engstrand

Radioactive Dating Laboratory

Stockholm 50, Sweden

$\mathrm{Su}$

FINLAND

Prof. Esa Hyyppä

Geological Survey of Finland

Otaniemi, Finland

T

TRONDHEIM

Mr. Reidar Nydal and Mr. Knut Lövseth

Radiological Dating Laboratory

The Norwegian Institute of Technology

Trondheim, Norway

TA TARTL

H. Simm or A. Liiva

Geobiochemistry Laboratory

Institute of Zoology and Botany Academy of Sciences of the Estonian SSR

Vanemuise St. 21

Tartu, Estonian, USSR

TAM TEXAS A \& M UNIVERSITY

Dr. Donald W. Hood

Dept. of Oceanography and Meteorology

Texas A \& M University

College Station, Texas 77843

TB

TBILISI

A. A. Burchuladze

Radiocarbon Laboratory

Tbilisi University

1 Chavchavadze Avenue

Tbilisi, USSR

*TBNC10 KAMAN NUCLEAR

Kaman Nuclear

Gardlen of the Gods Road

Colorado Springs, Colorado

TF

TATA INSTICUTE OF FUNDAMENTAL RESEARCH

Dr. D. Lal

Tata Institute of Fundamental Research

Homi Bhabha Road

Bombay-5 BR., India

TK

UNIVERSITY OF TOKYO

1)r. Hisashi Suzuki

Carbon Dating Laboratory

Department of Anthropology

Faculty of Science

University of Tokyo

Hongo, Bunkyo-ku, Tokyo, Japan

$\mathrm{Tx}$

TEXAS

Mr. S. Valastro, Jr. or Dr. E. Mott Davis

Radiocarbon Laboratory

Balcones Research Center, Rt. 4, Box 189

University of Texas at Austin

Austin, Texas 787.57 
U

CPJS.II.A

Dr. Ingrid Olssom

Institute of Physics

University of Uppsala

Uppsala, Sweden

CCLA UNIVERSITY OF CALIFORNIA, LOS ANGELES

Dr. Rainer Berger and Dr. W. F. Libby

Institute of Geophysics

University of California

Los Angeles, California 90024

LW INIVERSITY OF WASHINGTON

Dr. A. W. Fairhall

Department of Chemistry

University of Washington

Seattle, Washington 98105

VICTORIA

Anne Berminghan

Radiocarbon Dating Laboratory

Institute of Applied Science of Victoria

304-328 Swanston Street

Melbourne 3000, Australia

VRI VIENNA RADIUM INSTITUTE

1)r. H. Felber

Institut für Radiumforschung und Kernphysik

Boltzmanngasse 3

A-1090 Vienna, Austria

II I.S. GEOLOGICAL SURVEY

Dr. Meyer Rubin

U.S. Geological Survey

Washington, D.C. 20242

WIS WISCONSIN

Dr. Margaret Bender

Radiocarbon Laboratory of the Center for Climatic Research

Department of Meteorology

University of Wisconsin

Madison, Wisconsin 53706

WSU

WASHINGTON STATE UNIVERSITY

Dr. Roy M. Chatters

Radioisotopes and Radiations Laboratory

College of Engineering Research Division

Pullman, Washington 99163

$\mathrm{x}$

WHITWORTH COILEGE

Dr. Edwin A. Olson

Department of Geology

Whitworth College

Spokane, Washington 99218

Y

Y.II.E

Dr. Minze Stuiver

Radiocarbon Laboratory

Yale University

New Haven, Connecticut 06520 